\title{
Dynamic Research on China's Gini Coefficient
}

\author{
Xiao-hui HAI \\ School of Statistics and Mathematics, Inner Mongolia University of Finance and \\ Economics, Hohhot 010070, China
}

Keywords: Gini coefficient, Grouping weighting method, Income gap.

\begin{abstract}
Based on the grouped data of resident income in Statistical Yearbook, the paper calculates the China's Gini coefficients of urban, rural and national residents in the ten years from 2002 to 2011 according to the characteristics of income data of urban residents and rural residents, and finally analyzes the Gini coefficients of urban, rural and national residents calculated, to come to the conclusion that the Gini coefficient of resident income breaks through the warning line and keeps highly stable, which reflects the wealth gap of China's residents in the current stage is still in the process of developing from a rational gap to an excessive gap, but there is no polarization.
\end{abstract}

\section{Introduction}

China has gradually entered the stage of a wealthy and strong country since the reform and opening up, but there is no doubt that the gap of resident income also becomes larger. Gini coefficient gives the quantity line reflecting the economic difference among residents, and based on that, the wealth gap among residents can be reflected intuitively and objectively, to provide early warning and prevent the appearance of wealth polarization, and it is an internationally accepted authoritative index measuring the level of a country's wealth gap and income distribution gap. The paper studies the dynamic trajectory of Gini coefficient through calculating the Gini coefficients of China in recent ten years, so as to grasp the distribution situation and variation trend of China's resident income.

\section{The Empirical Analysis on Gini Coefficient}

\section{The Computing Method of Gini Coefficient}

As a common conception measuring income gap, Gini coefficient descries the comparative deviation degree of average income gap caused by population distribution to the expected value of total income. Its computing method is based on the data which evenly distribute population into several groups $\mathrm{N}$, namely the proportion of each group's population to the total population is the same, and meanwhile, the mean value $\mu$ of corresponding evenly divided group can be obtained, so the computing formula of Gini Coefficient is $G=\frac{1}{2 \mu N^{2}} \sum_{i=1}^{N} \sum_{j=1}^{N}\left|y_{i}-y_{j}\right|$.

$\left|y_{i}-y_{j}\right|$ represents the absolute value of the income mean difference of any two evenly divided groups. $\mu$ represents the expected value of various evenly divided groups' total income. According to the formula, it can be found that $\mathrm{G}$ is the average deviation of total income. The average deviation value will be divided by the expected value of total income $\mu$ to obtain the comparative deviation degree of average income deviation to the expected value of total income $\mu$, which is Gini coefficient $\mathrm{G}$. 


\section{The Calculation of Urban Gini Coefficient}

The mean values of urban resident income in different groups are determined according to the distribution of population, but due to the unequal grouping proportion of population as well as the difference in the number of members in each household in corresponding groups, the income mean values of several groups are determined not based on the grouping of equal population proportion. Therefore, the problem needing to be solved is to regroup based on the existing information and work out the corresponding income mean values of those groups according to the several evenly divided groups of population. For the specific computing process, it can be carried in accordance with the following two steps.

Firstly, according to the characteristics of 7 groups of values given in the Statistical Yearbook, they can be preliminary divided into 5 groups which are based on approximately even division; and then according to the proportions of household groups and their corresponding population data of each household, the corresponding proportion values of those 7 groups can be calculated.

Secondly, implementing even distribution for the population in the groups whose population proportion closing to $20 \%$, to realize that the population proportions of all five groups equal to $20 \%$, and work out the corresponding income mean value in various groups.

Based the first assumption, the corresponding values of point 10, 30, 50, 70, 90 and other points can be calculated to regard them as the income mean values of those five evenly divided groups, see Table 1.

Table 1: The Mean of the Five Evenly Divided Groups' Corresponding Income

\begin{tabular}{c|c|c|c|c|c}
\hline Group & One & Two & Three & Four & Five \\
\hline $\begin{array}{c}\text { Evenly Divided } \\
\text { Population Distribution }\end{array}$ & 20 & 20 & 20 & 20 & 20 \\
\hline $\begin{array}{c}\text { Corresponding Income } \\
\text { Mean Value }\end{array}$ & 3510.9 & 5736.55 & 7868.7 & 10532.08 & 19225.77 \\
\hline
\end{tabular}

According to the corresponding income mean value of those evenly divided groups, we can work out that $\sum_{i=1}^{N} \sum_{j=1}^{N}\left|y_{i}-y_{j}\right|=72443.86$, and the overall mean value of income in those five groups $\mu=9373.86$. Based on $N=5$, various values are brought into the formula to obtain the urban resident coefficient in $\mathrm{G}_{2004}=0.309131$.

\section{The Calculation of Rural Gini Coefficient}

The calculation method of rural Gini coefficient is completely the same as that of urban Gini coefficient, and the difference between them is the data of urban resident data are evenly divided into 5 groups, see the following Table 3.

In a similar way, according to the data, we can work out that $\sum_{i=1}^{N} \sum_{j=1}^{N}\left|y_{i}-y_{j}\right|=25424.60$, and the mean value $\mu=2996.7$. Based on $N=5$, various values are brought into the formula to obtain the urban rural coefficient in $\mathrm{G}_{2004}=0.339368$. 


\section{The Calculation of National Gini Coefficient}

The Gini coefficient for the national resident income can be obtained based on the income Gini coefficients of China's urban residents and rural residents through using "grouping weighting method".

Here, $G_{c}$ and $G_{r}$ represent the Gini coefficient of urban resident income differences and rural resident income differences respectively, $\mathrm{P}_{\mathrm{c}}$ and $\mathrm{P}_{\mathrm{r}}$ represent urban and rural population proportion respectively, $\mathrm{u}_{\mathrm{c}}$ and $\mathrm{u}_{\mathrm{r}}$ represent urban and rural per capita income respectively, and $\mathrm{u}$ represents the national per-capita income.

Based on that, the Gini coefficient of national residents obtained is $\mathrm{G}=0.424269126$

Through using the same method, the Gini coefficients of rural, urban and national resident income in the period from 2001 to 2011 can be obtained, and for specific results, see Table 2.

Table 2: The Gini Coefficient of Chinese Residents From 2002 to 2011

\begin{tabular}{cccc}
\hline Year & Urban Gini Coefficient & Rural Gini Coefficient & National Gini Coefficient \\
\hline 2002 & 0.294169 & 0.340837 & 0.409472666 \\
2003 & 0.301296 & 0.349663 & 0.420866062 \\
2004 & 0.309131 & 0.339368 & 0.424269126 \\
2005 & 0.315221 & 0.345228 & 0.430693921 \\
2006 & 0.312163 & 0.344003 & 0.431311226 \\
2007 & 0.309288 & 0.343895 & 0.430460713 \\
2008 & 0.314817 & 0.347698 & 0.434186574 \\
2009 & 0.311077 & 0.355127 & 0.43363104 \\
2010 & 0.30557 & 0.348922 & 0.422865638 \\
\hline
\end{tabular}

\section{The Changing Tendency of China's Gini Coefficient}

\section{The Changing Tendency of Rural and Urban Gini Coefficients and China's Gini Coefficient}

With the rapid and sustaining growth of economy as well as the large-scale change of systems, the income gap of Chinese residents expands continuously, which is shown in the all-round expansion of urban resident income gap, rural resident income gap and national resident income gap. Table 3 shows the data comparison among the Gini coefficients of China's rural, urban and national resident income, therefore, the three characteristics about the changes in China's resident income gap can be obtained.

The income gap between rural and urban residents basically shows a stable tendency, but their change trajectories are different. On the whole, the Gini coefficient of rural resident income is higher than the Gini coefficient of urban resident income.

The fluctuations in the Gini coefficient of China's national resident income can be generally divided into two stages. The first stage is from 2002 to 2008, and the Gini coefficient of this stage basically shows a tendency of growth, reaching the peak 0.434 in 2008 . The second stage is the last three years, the Gini coefficient decreases continuously, and the descend range in the period from 2009 to 2010 is the biggest, decreasing by 2.48 percent. On the whole, the national resident income gap is in a high level of stale stage. 
The rural and urban income gap is the main source of China's national resident income gap. The Gini coefficient of national resident income is always higher than the Gini coefficients of rural and urban resident income, which is above the rural and urban Gini coefficients, and it shows the income gap between rural and urban areas is an important source of national resident income gap. What's more, the variation in the Gini coefficient of China's national resident income and the variation in the Gini coefficients of rural and urban resident income are basically synchronous, so the substantial correlation between China's national resident income gap and rural and urban resident income gap means that the rural and urban income gap is the main source of China's national resident income gap, which even affects the change tendency of China's national resident income gap.

Table 3 The Gini coefficients of China's rural, urban and national resident income,

\begin{tabular}{c|c|c|c}
\hline & Ruralm Gini coefficient & Urban Gini coefficient & Urban Gini coefficient \\
\hline 2002 & 0.2942 & 0.3408 & 0.4095 \\
\hline 2003 & 0.3013 & 0.3497 & 0.4209 \\
\hline 2004 & 0.3091 & 0.3394 & 0.4243 \\
\hline 2005 & 0.3152 & 0.3452 & 0.4307 \\
\hline 2006 & 0.3122 & 0.344 & 0.4313 \\
\hline 2007 & 0.3093 & 0.3439 & 0.4305 \\
\hline 2009 & 0.3148 & 0.3477 & 0.4342 \\
\hline 2010 & 0.3111 & 0.3551 & 0.4336 \\
\hline 2011 & 0.3056 & 0.3489 & 0.4229 \\
\hline
\end{tabular}

\section{Conclusion}

The paper uses the grouping data about the rural and urban resident income in the statistical yearbook released by National Bureau of Statistics of China to work out the Gini coefficients of rural and urban resident income in the period from 2004 to 2011, and uses the grouping weighting method to calculate the Gini coefficient of China's national resident income. Then those Gini coefficients are compared with the Gini coefficient released by China, and the results show that the overall changing law of the Gini coefficients calculated in the paper is basically the same as the Gini coefficients released by China. For the specific changing law, see the following part:

a. The Gini coefficient of China's rural, urban and national resident income has maintained stable on the whole since 2002, showing a down trend;

b. The Gini coefficient of China's national resident income is higher than the international warning line in the period from 2002 to 2011;

c. The Gini coefficient of China's national resident income is always higher than the Gini coefficient of rural and urban resident income;

d. The variation in the Gini coefficient of China's national resident income and the variation in the Gini coefficients of rural and urban resident income are basically synchronous. 
Indeed, decision-makers should pay attention to the situation that the Gini coefficient of resident income breaks through the warning line and maintains a high level of stability. However, they also should have correct understanding about the expanding wealth gap among China's residents and have scientific judgment about the wealth gap among residents in the turning period. The Gini coefficient of China's national resident income is always higher than the Gini coefficient of rural and urban resident income, and the variation in the Gini coefficient ofChina's national resident income and the variation in the Gini coefficients of rural and urban resident income are basically synchronous, which reflects the income gap between rural and urban areas is an important source of China's national resident income gap, so it shows that controlling rural and urban resident income gap can controlling the Gini coefficient of China's national resident income well. Through measuring the wealth gap among China's residents in the current stage by using the Gini coefficients calculated in the paper, the result shows the wealth gap of China's residents in the current stage is still in the process of developing from a rational gap to an excessive gap, but there is no polarization. Objectively, since 1990s, the national economy of China has shown a state of sustaining and healthy development, the comprehensive strength has been enhanced continuously, the income level and life quality of residents has improved continuously, and the consumption structure of rural and urban residents is optimized continuously. Therefore, the wealth gap of China in the current stage is a relative difference rather than absolute difference, and the gap is based on the sustaining and rapid development of China's national economy. The wealth gap caused in the process that the overall income level of people is generally improved, refers to the difference of being rich earlier or later under the target of collective prosperity, and it does not include the content of polarization.

\section{References}

[1] Jiqiang Guo: submitted to Social Sciences in Chinese (2011).

[2] César Ducrute: submitted to Chinese Geographical Science (2012).

[3] Jinzhong Lin, Ying Wang: submitted to Economist in Chinese (2007).

[4] Shuang Peng, Xiaodong Ye: submitted to Economist in Chinese (2008).

[5] Shi Li: submitted to Social Sciences in Chinese (2000). 